\title{
Practical and Scalable Manufacturing Process for Plasma Kallikrein Inhibitor ASP5069
}

\author{
Shun Hirasawa* and Yoshinori Kohmura \\ Pharmaceutical Science \& Technology Labs., Pharmaceutical Technology, Astellas Pharma Inc.,
}

21 Miyukigaoka, Tsukuba-shi, Ibaraki 305-8585, Japan

\section{Supporting Information}

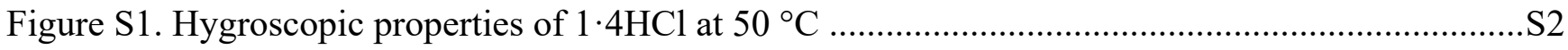

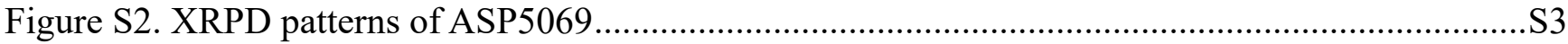

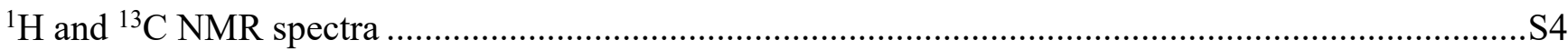


Figure S1. Hygroscopic properties of $1 \cdot 4 \mathrm{HCl}$ at $50{ }^{\circ} \mathrm{C}$

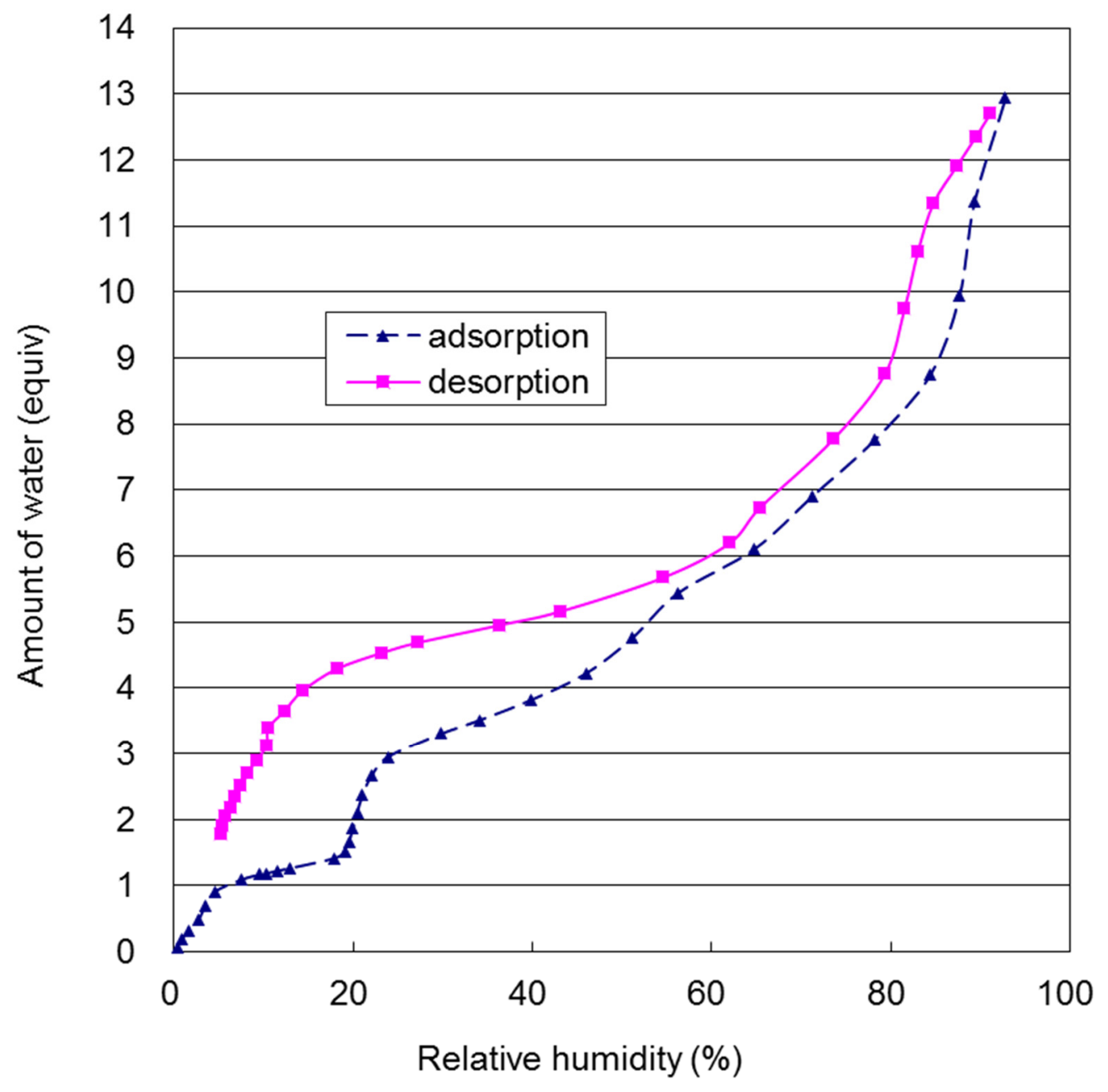


Figure S2. XRPD patterns of ASP5069

(a) Desired polymorph

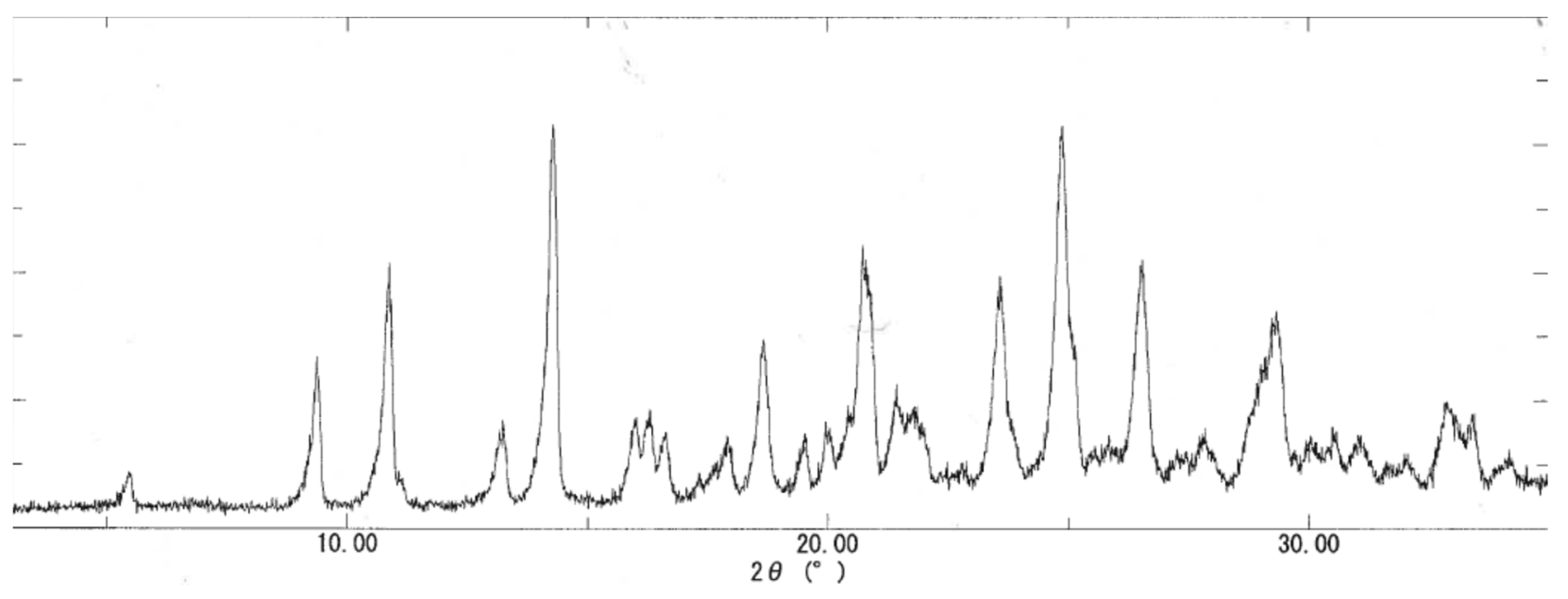

(b) Contamination with an undesired polymorph or pseudopolymorph at $\mathrm{pH} 6.8$ (red circle)

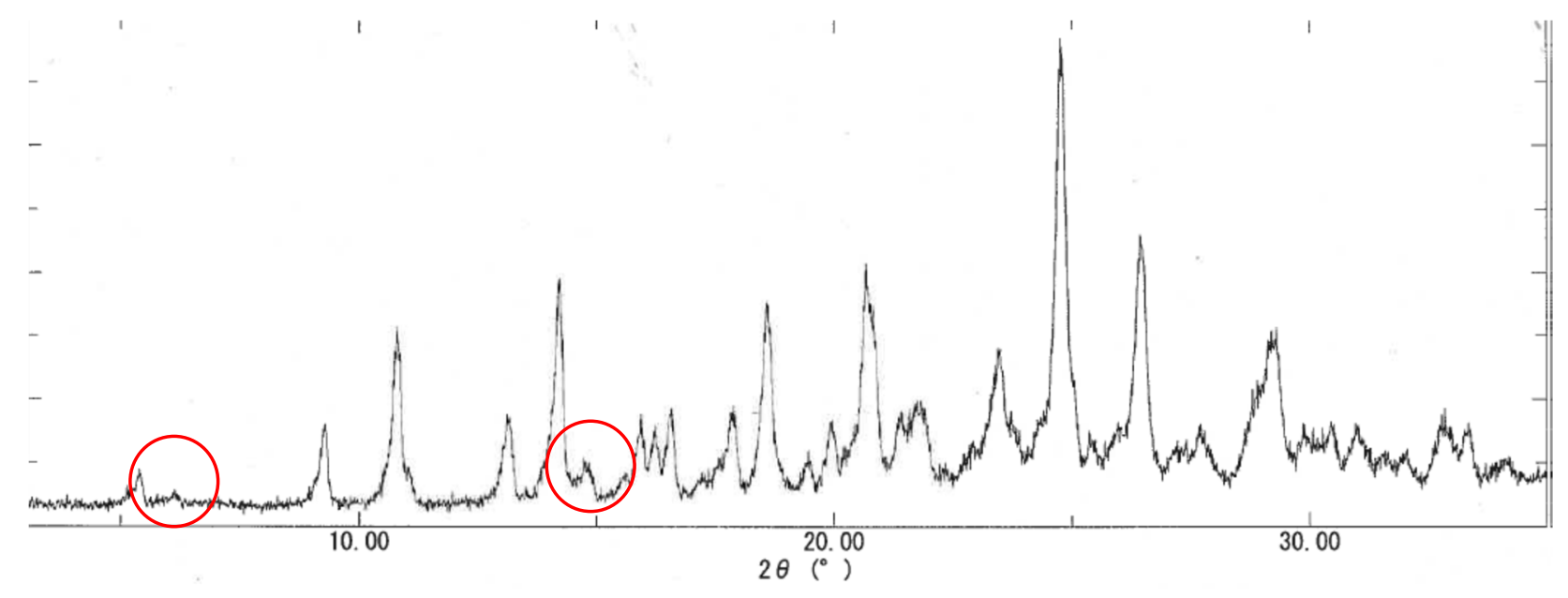




\section{${ }^{1} \mathrm{H}$ and ${ }^{13} \mathrm{C}$ NMR spectra}

Compound 3

${ }^{1} \mathrm{H}$ NMR (DMSO-d6, $500 \mathrm{MHz}$ )
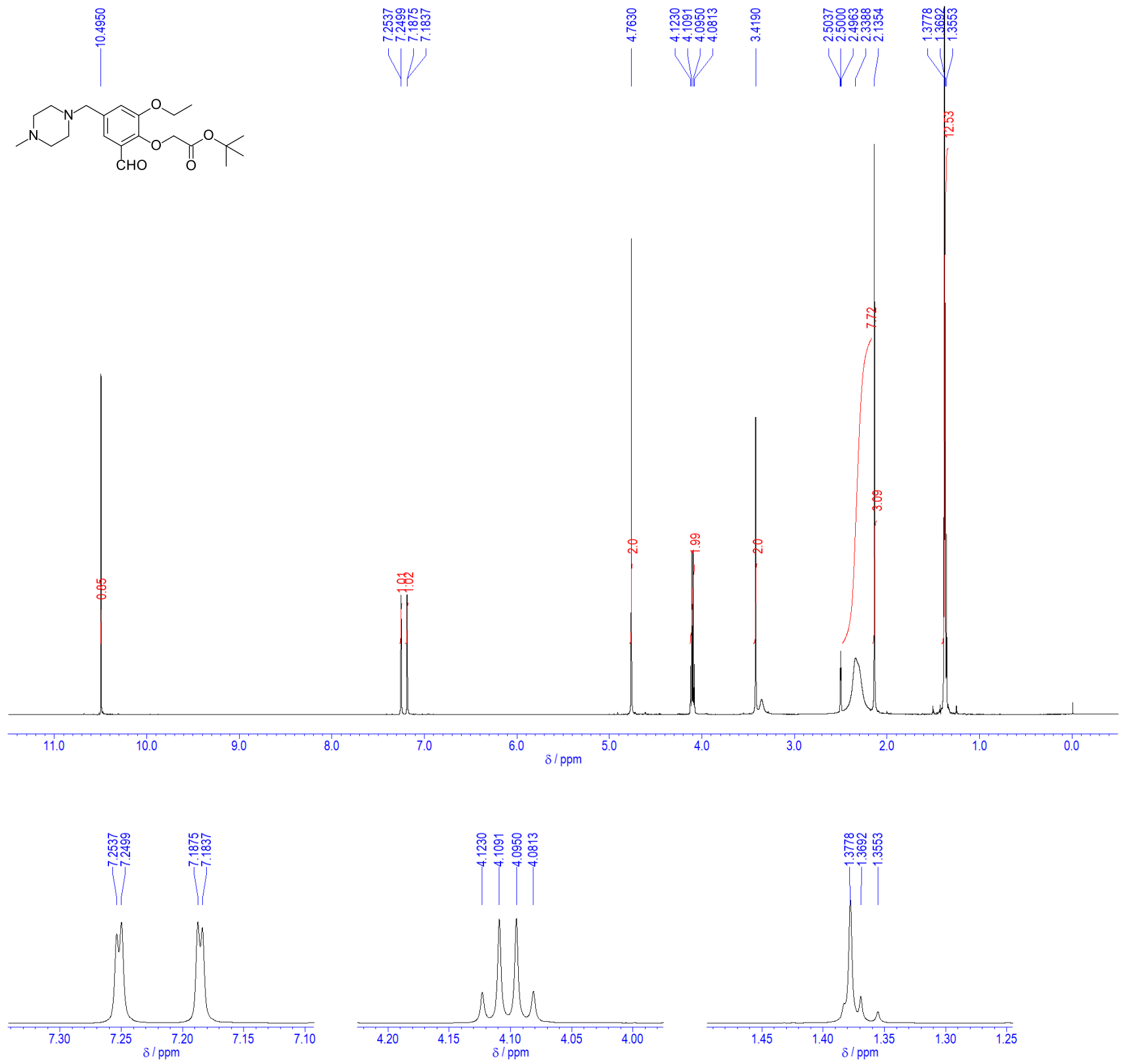


\section{Compound $\mathbf{3}$}

${ }^{13} \mathrm{C}$ NMR (DMSO- $d_{6}, 125 \mathrm{MHz}$ )
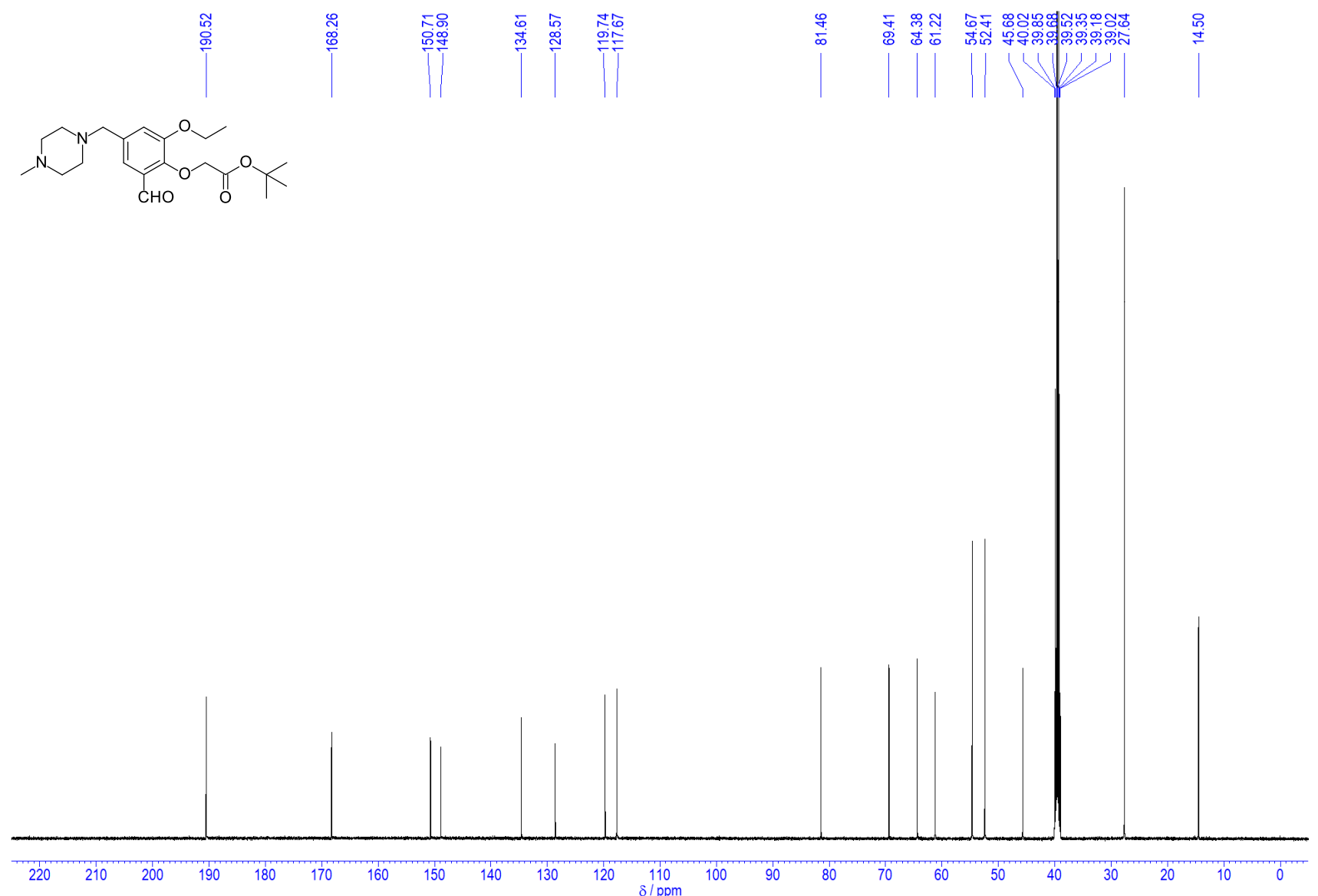


\section{Compound 8}

${ }^{1} \mathrm{H}$ NMR (DMSO- $d_{6}, 500 \mathrm{MHz}$ )

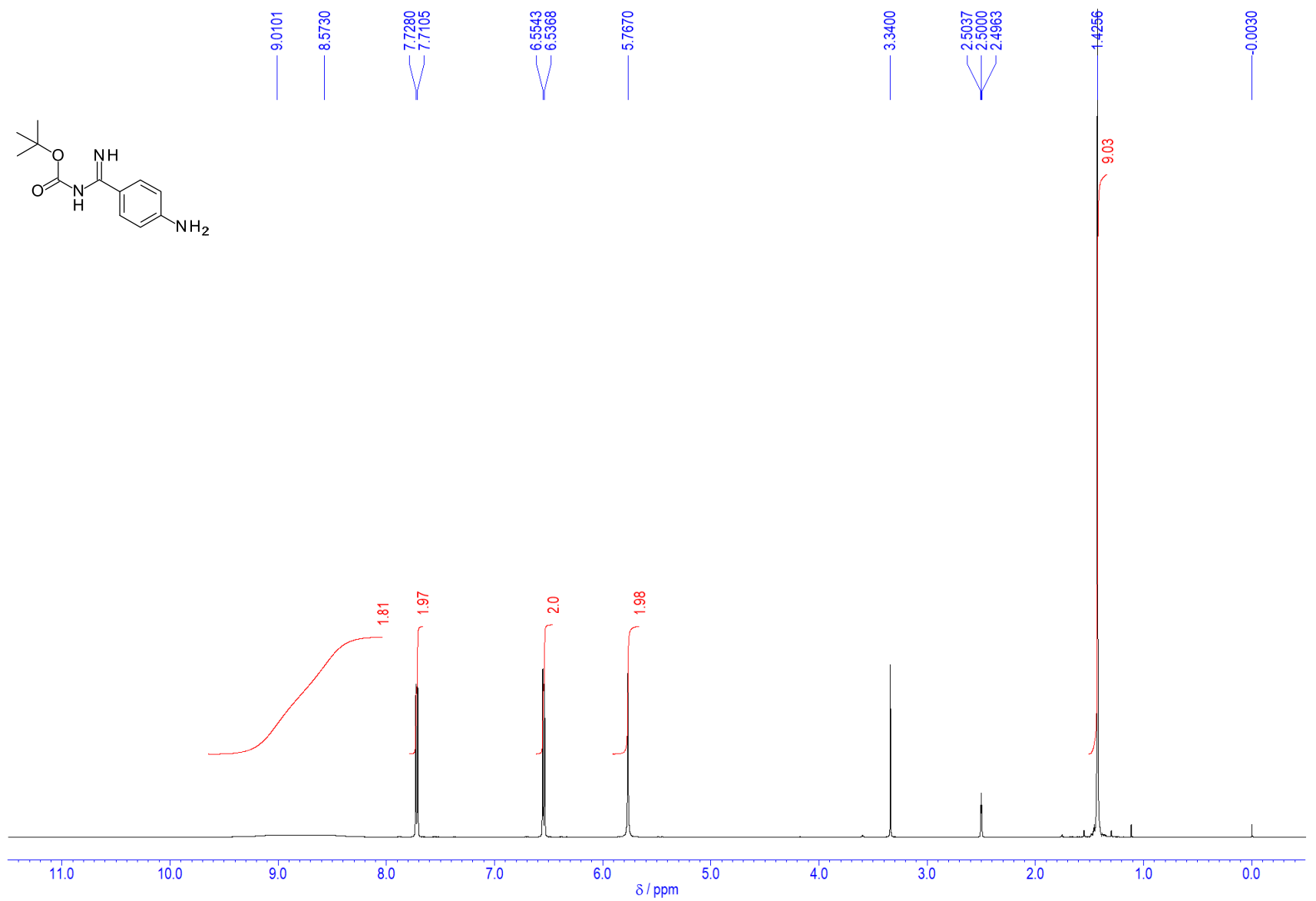




\section{Compound 8}

${ }^{13} \mathrm{C}$ NMR (DMSO- $d_{6}, 125 \mathrm{MHz}$ )
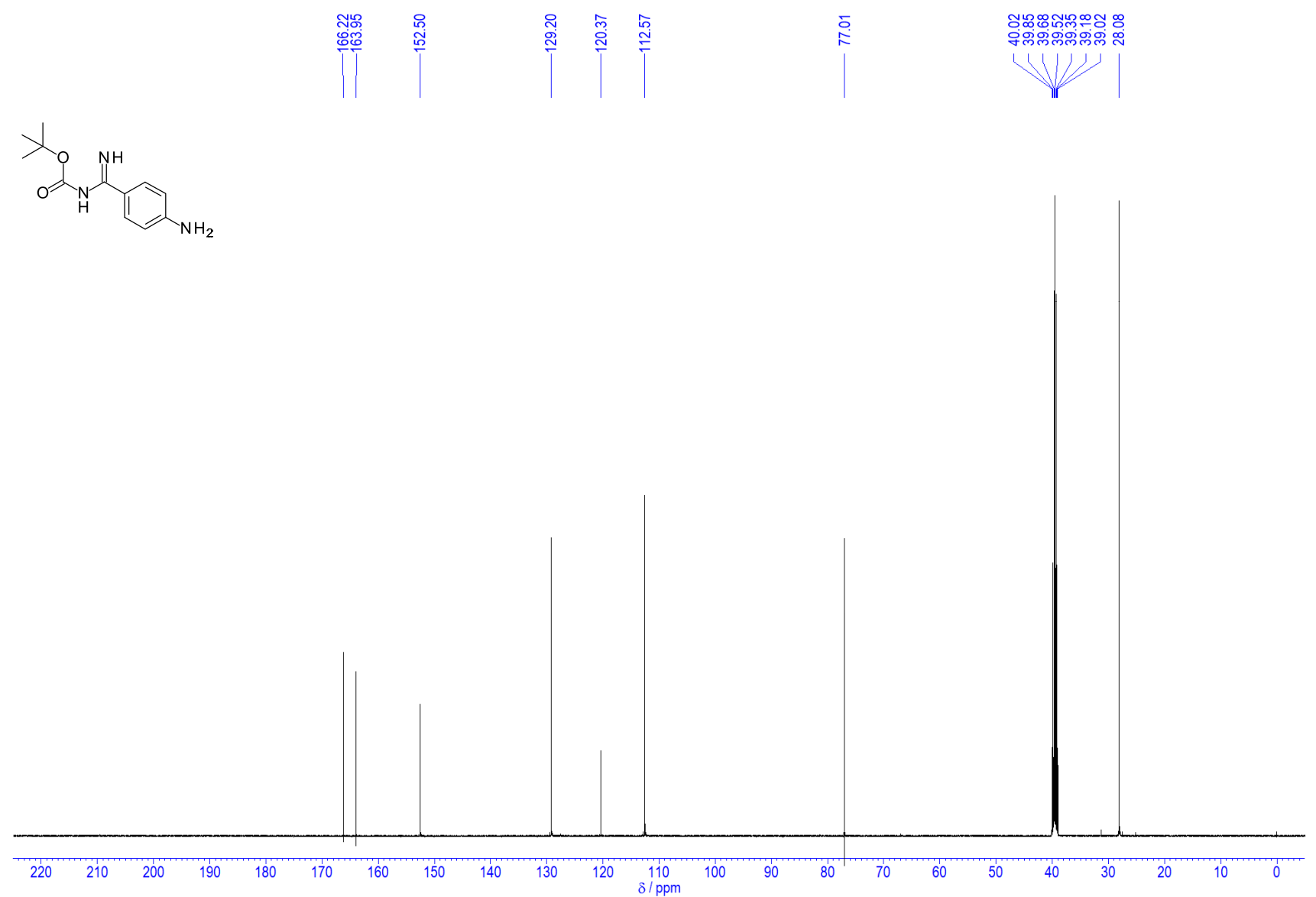


\section{Compound $\mathbf{1 0}$}

${ }^{1} \mathrm{H}$ NMR (DMSO- $d_{6}, 500 \mathrm{MHz}$ )
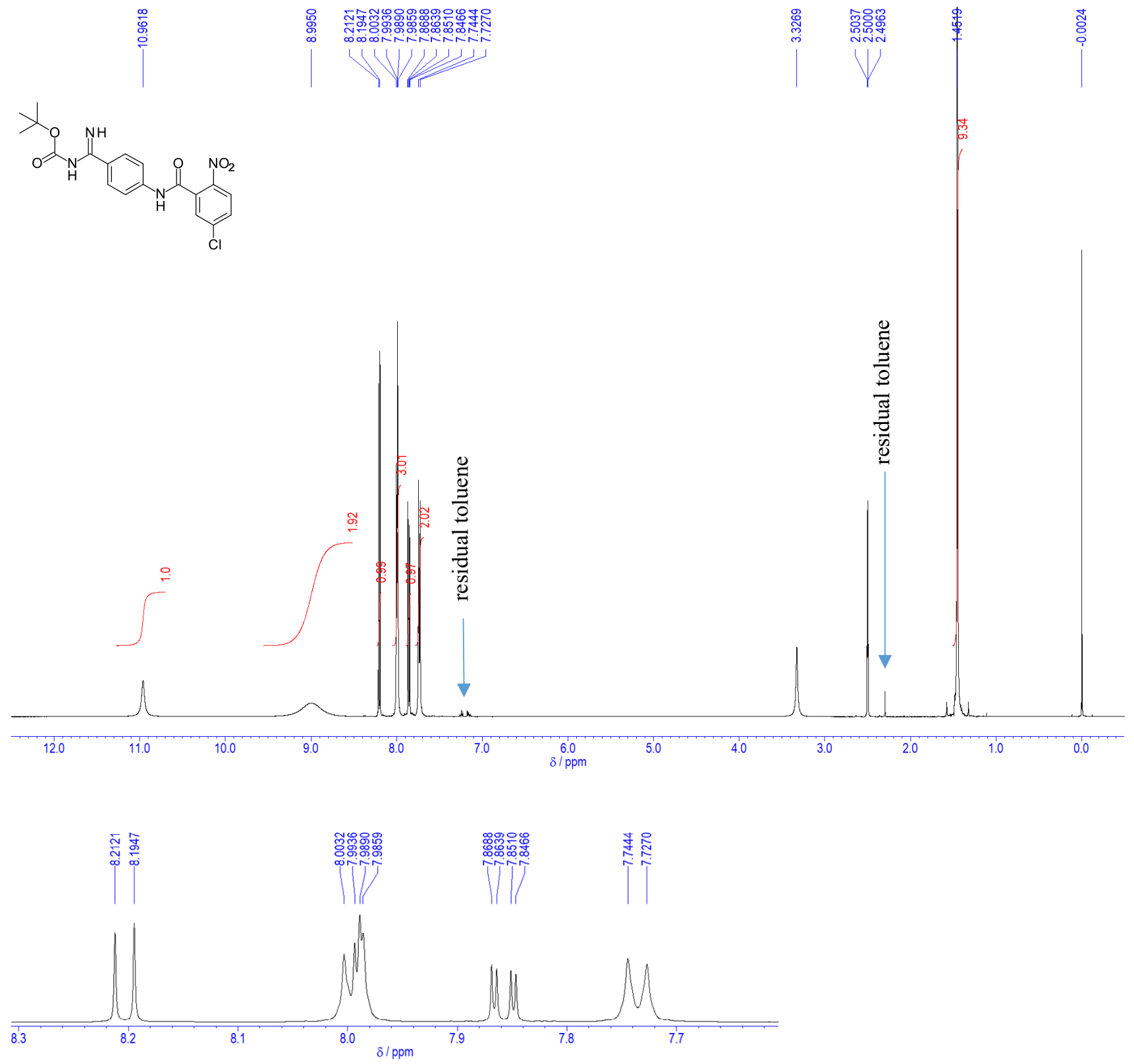


\section{Compound 10}

${ }^{13} \mathrm{C}$ NMR (DMSO- $d_{6}, 125 \mathrm{MHz}$ )

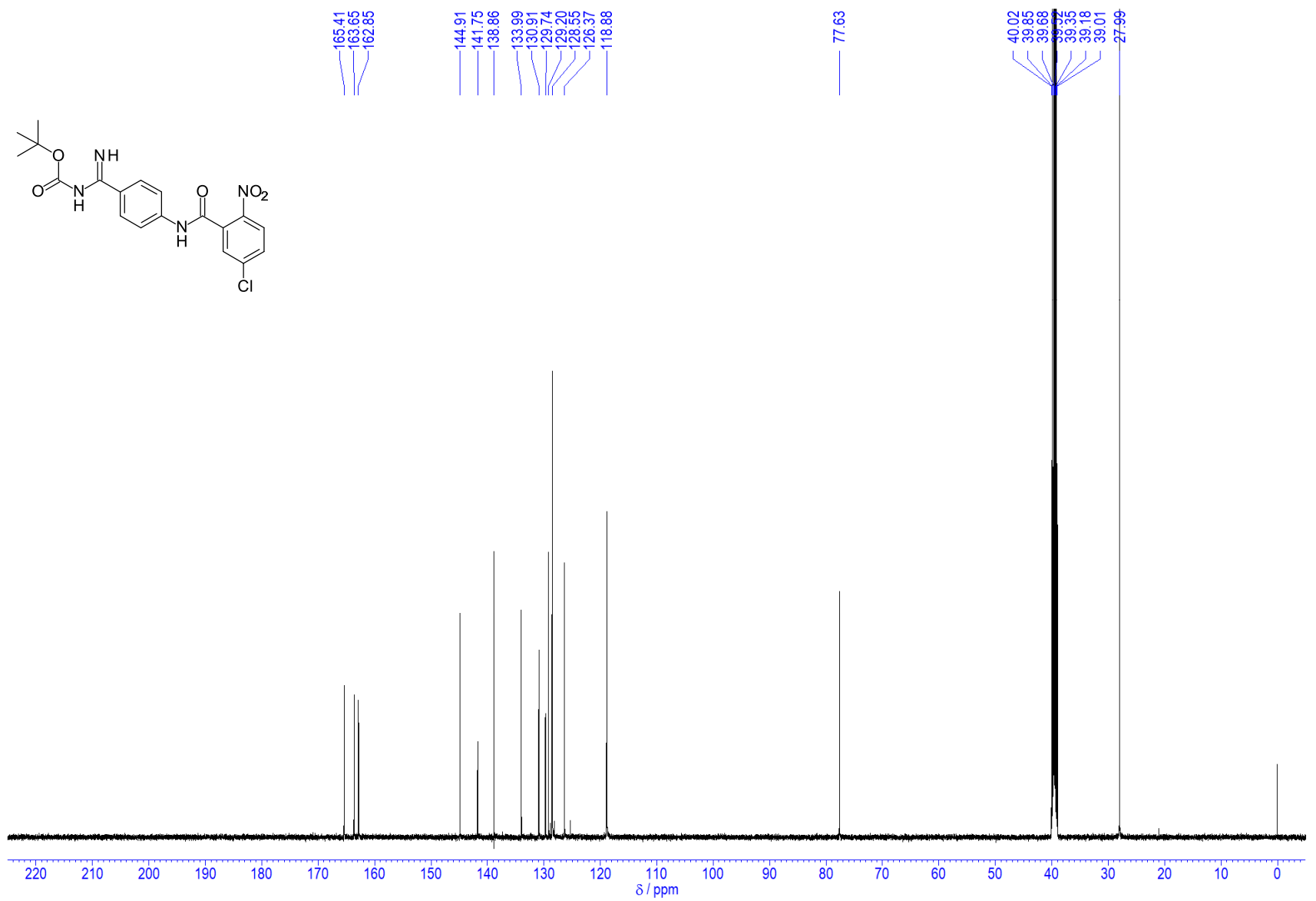




\section{Compound 5}

${ }^{1} \mathrm{H}$ NMR (DMSO- $d_{6}, 500 \mathrm{MHz}$ )
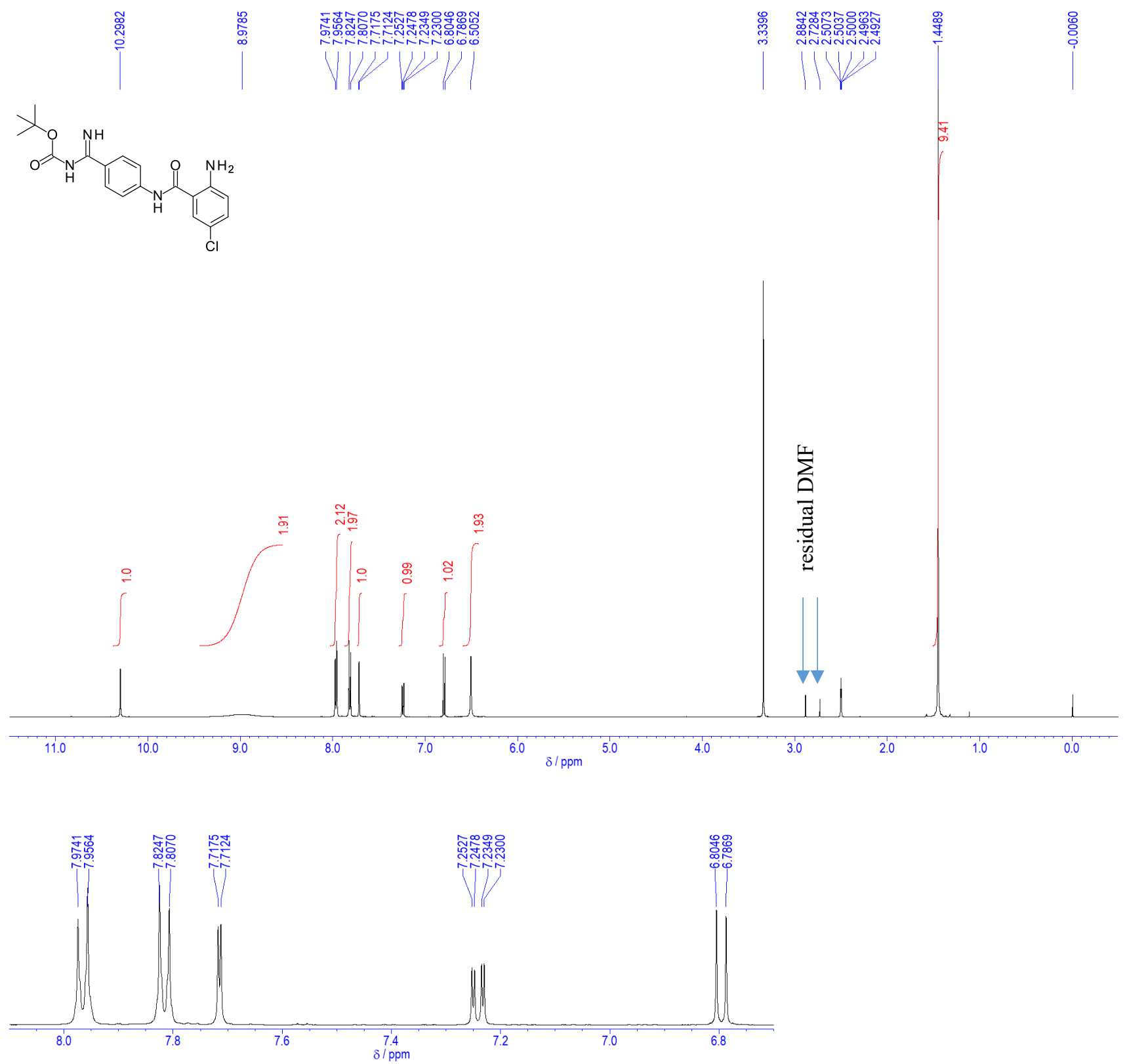


\section{Compound 5}

${ }^{13} \mathrm{C}$ NMR (DMSO- $d_{6}, 125 \mathrm{MHz}$ )

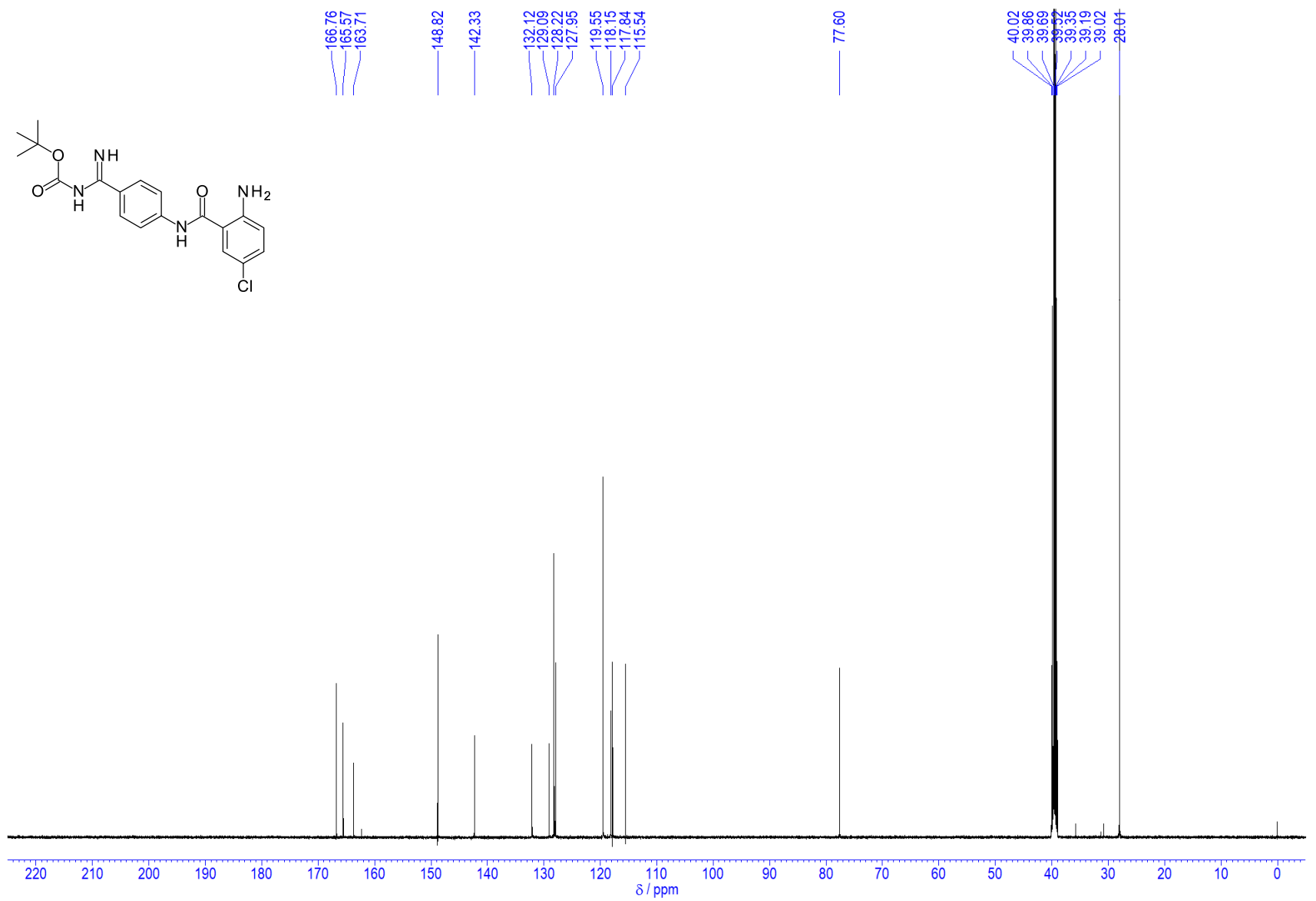




\section{Compound 11}

${ }^{1} \mathrm{H}$ NMR (DMSO- $d_{6}, 500 \mathrm{MHz}$ )
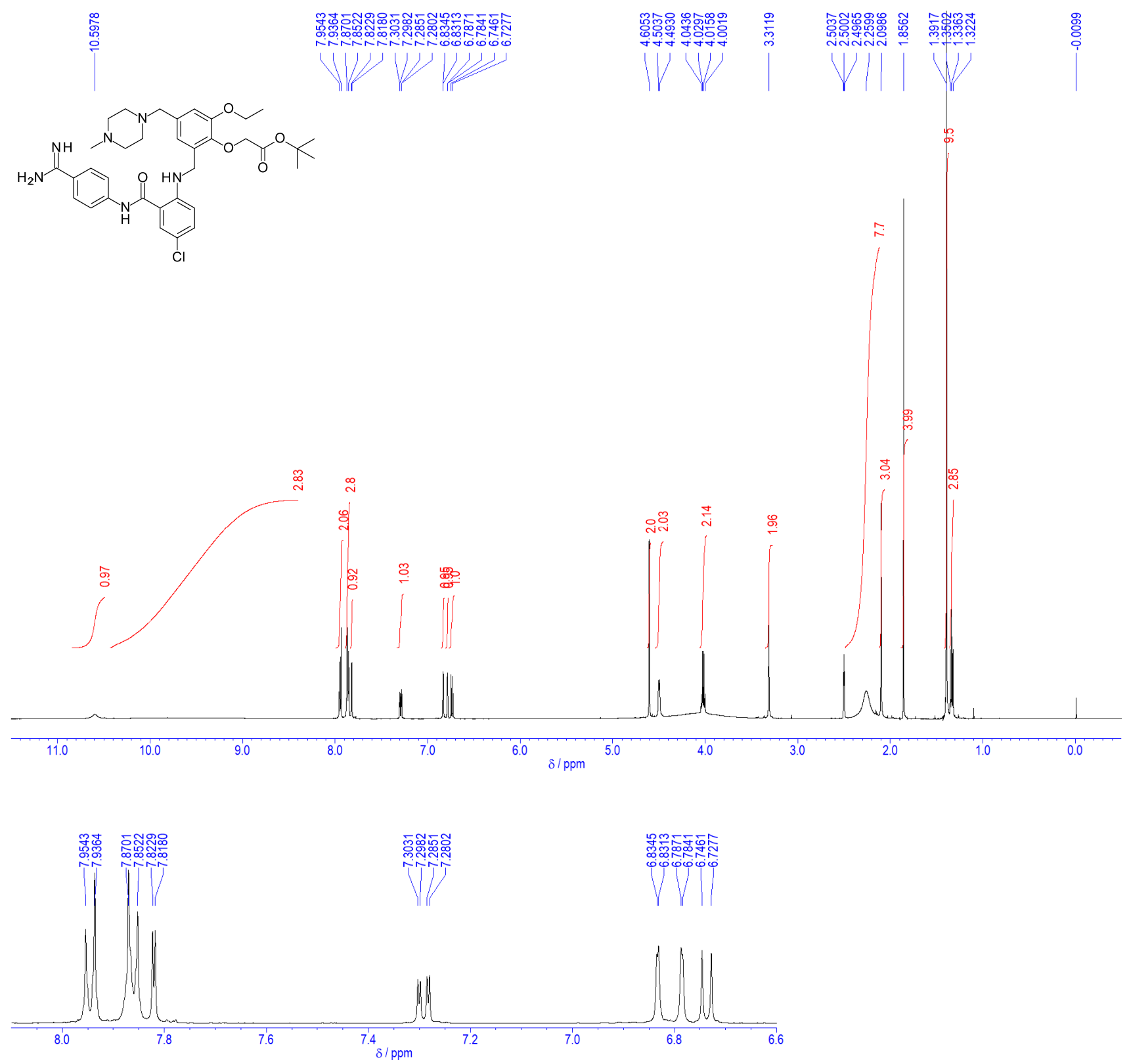


\section{Compound 11}

${ }^{13} \mathrm{C}$ NMR (DMSO- $d_{6}, 125 \mathrm{MHz}$ )

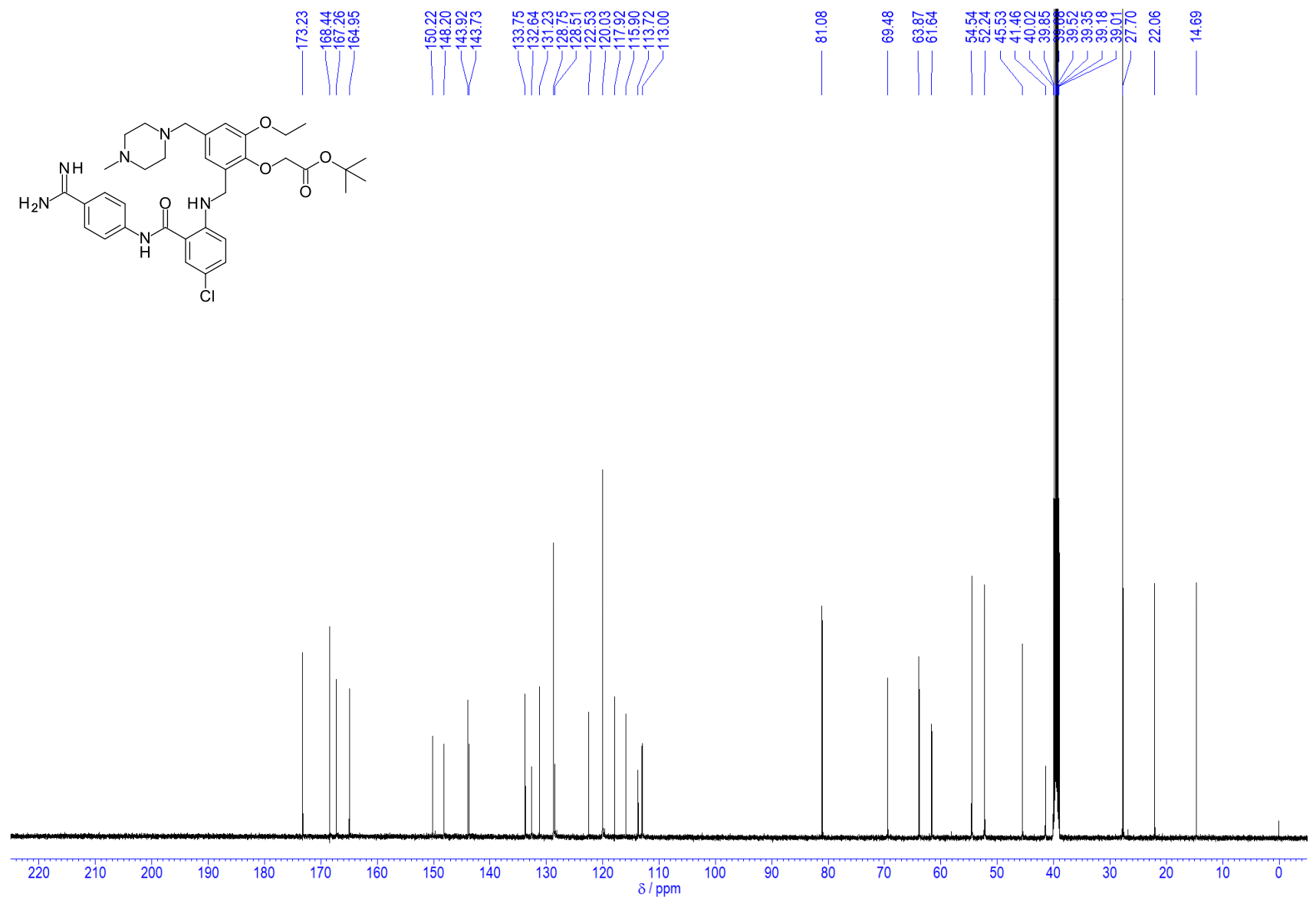


$\operatorname{ASP} 5069\left(\mathbf{1} \cdot \mathrm{HCl} \cdot 2 \mathrm{H}_{2} \mathrm{O}\right)$

${ }^{1} \mathrm{H}$ NMR (DMSO-d6, $500 \mathrm{MHz}$ )
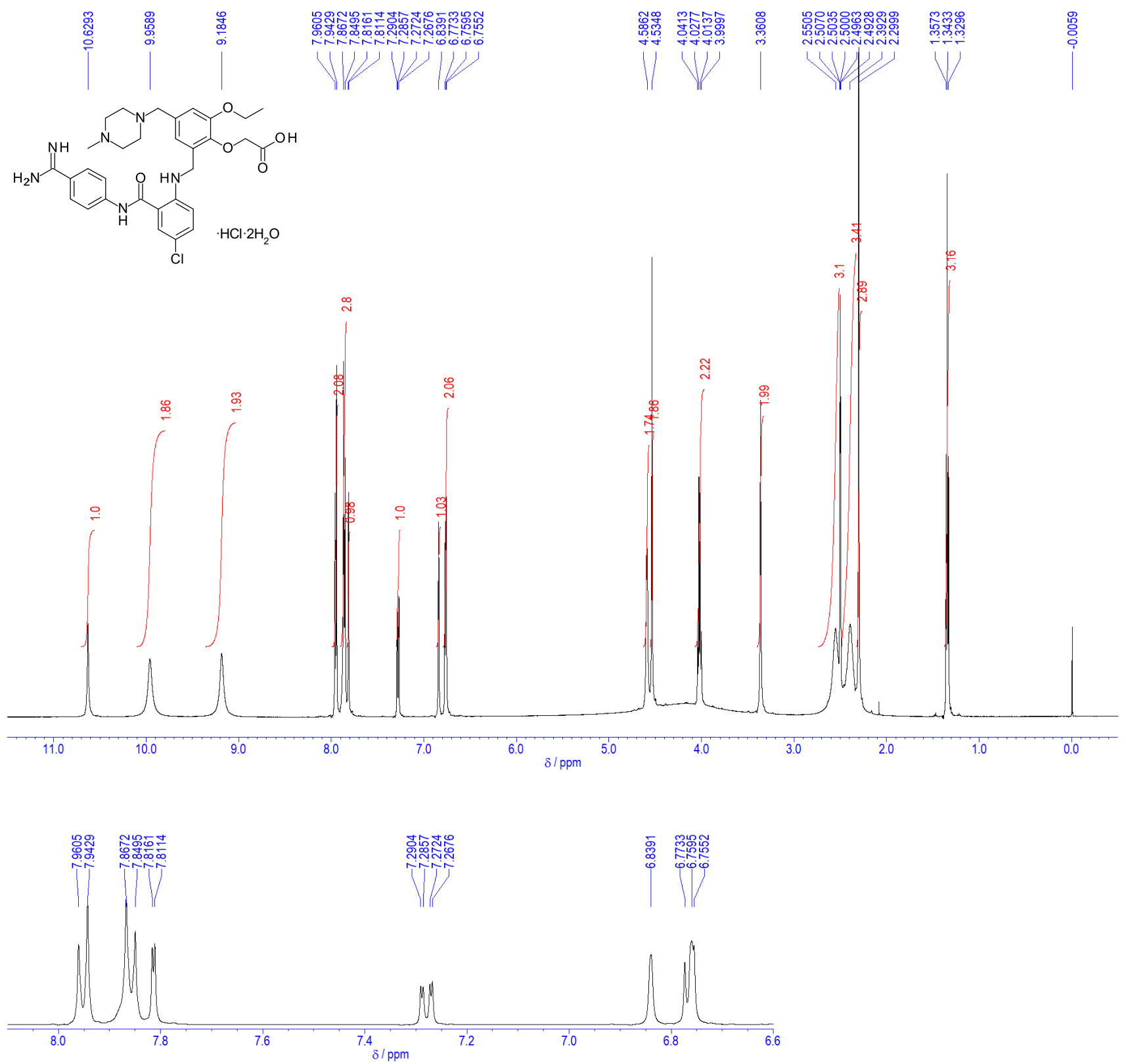
$\operatorname{ASP} 5069\left(\mathbf{1} \cdot \mathrm{HCl} \cdot 2 \mathrm{H}_{2} \mathrm{O}\right)$

${ }^{13} \mathrm{C}$ NMR (DMSO- $d_{6}, 125 \mathrm{MHz}$ )

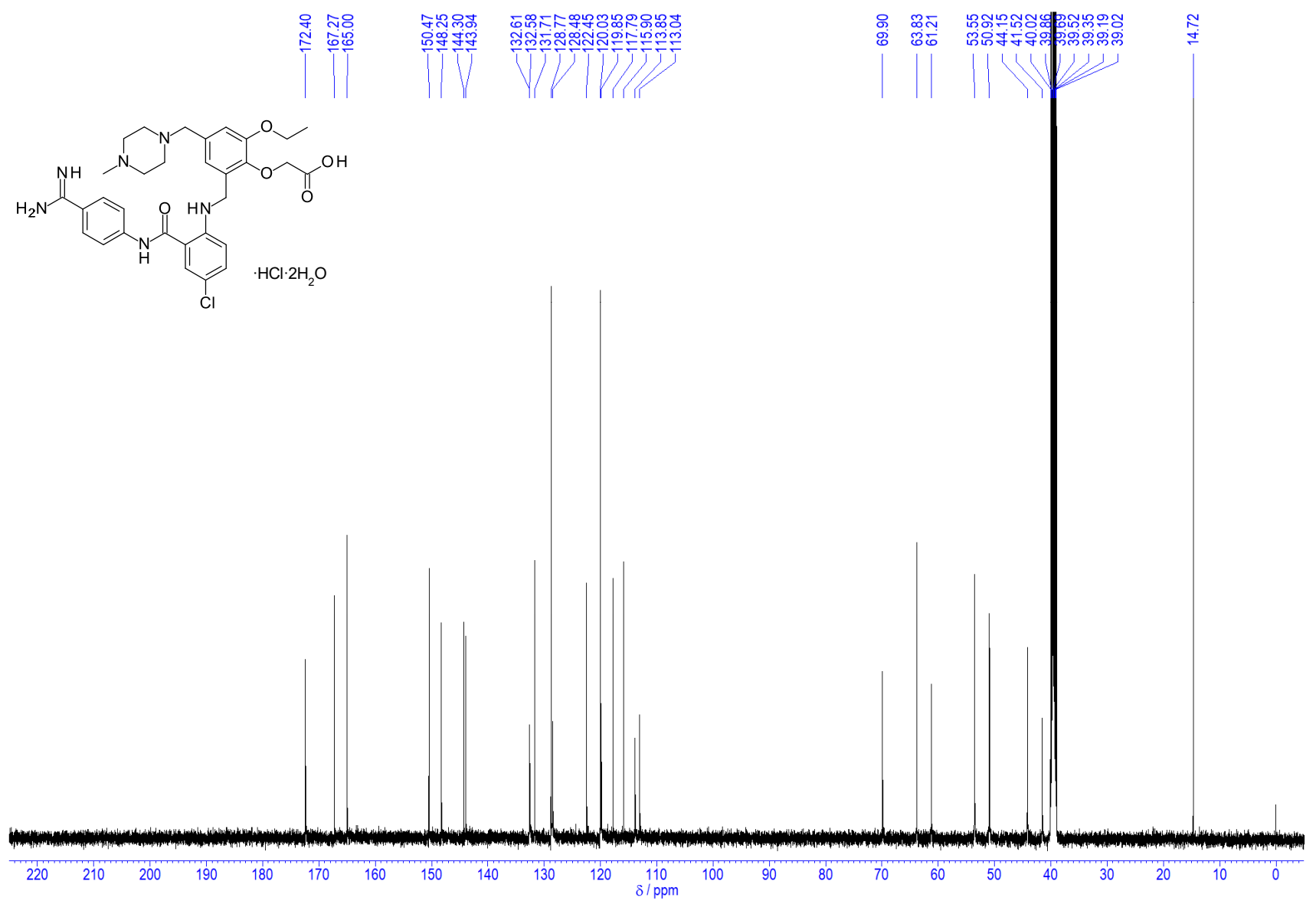

